\title{
An industry update: the latest developments in the field of therapeutic delivery
}

First draft submitted: 21 April 2017; Accepted for publication: 11 May 2017; Published online: 21 June 2017

Keywords: acquisitions and mergers $\bullet$ clinical trials $\bullet$ pipeline news

March 2017, the period covered by this article, was characterized by a number of interesting acquisitions and mergers in a period when, surprisingly, merger and acquisition activity was being reported as low, at least as being down from the same period in 2016 [1]. From a 'big pharma' perspective, the month saw Johnson and Johnson's (NJ, USA) acquisition of Actelion (Allschwil/Basel, Switzerland) moving to a completion, Lilly acquiring CoLucid (MA, USA) and taking back an asset they had originally licensed to CoLucid, and Otsuka (Tokyo, Japan) acquired Neurovance. Smaller companies, including Tessa Therapeutics (Singapore) and Euchloe Bio (Singapore), Threshold Pharmaceuticals and Molecular Templates (TX, USA), came together to form larger entities. Advances in company pipelines were disclosed in several international meetings held in March, including those of the American Academy of Dermatology (AAD; FL, USA), the Annual Maui Derm for Dermatologists (HI, USA) and the American College of Cardiology (ACC) Annual Scientific Meeting (DC, USA). Information from these meetings provides for most of the last section of this article on updates of therapeutic advances from clinical trials. Also several technologies enabling delivery of therapeutic agents figured in the news in March, including the virus-specific T-cell technology gained by Tessa Therapeutics with their acquisition of Euchloe Bio, a GalNAc-conjugated RNAi therapeutic being developed jointly by The Medicines
Company (NJ, USA) and Alnylam Pharmaceuticals (MA, USA), the agreement made between Heptares and Daiichi Sankyo giving the latter access to the $\mathrm{STaR}^{\circledR}$ stabilized G-protein coupled receptor (GPCR) technology, the release of Phase Ib clinical trial data on an Ablynx (Ghent, Belgium) Nanobody ${ }^{\circledR}$ therapeutic protein by partner Merck KgaA and the advancement of CytomX's Probody ${ }^{\circledR}$ platform technology in an agreement with Bristol-Myers Squibb (NY, USA).

\section{Business news}

\section{Acquisitions, mergers}

\section{Johnson \& Johnson \& Actelion}

The very end of 2016 saw the start of the engagement between Johnson and Johnson and Actelion in respect of an acquisition of the Swiss biopharmaceutical company by the US healthcare giant. This has reached a conclusion with the announcement at the end of March by the Swiss subsidiary of Johnson and Johnson, Janssen Holding GmbH of the successful tender offer for Actelion [2]. The acceptance period for the tender offer was set to expire on 21 April and as the antitrust clearances were already completed, the settlement is expected to occur during the second quarter of 2017. As part of the transaction, Actelion will spin out its drug discovery and early clinical development assets into a new Swiss biopharmaceutical company, Indorsia Ltd. In addition to gaining the approved and
Peter Timmins

School of Pharmacy, University of Huddersfield, Huddersfield HD1 3DH, UK p.timmins@hud.ac.uk 
late development assets of Actelion, Johnson and Johnson will hold $16 \%$ of Indorsia shares with a right to potentially increase its holding to $32 \%$.

\section{Lilly \& CoLucid Pharmaceuticals}

CoLucid Pharmaceuticals licensed the selective 5-HT1F serotonin receptor agonist lasmiditan (COL144) from Eli Lilly (IN, USA) in 2006. CoLucid undertook its development in the treatment of acute migraine, where it may differentiate from the standardof-care triptans by its poorer affinity for 5-HT1B and 5-HT1D receptors that triptans additionally bind to, and that may be associated with some of the side effects of triptans. On the back of a late 2016 successful Phase III trial, Lilly have now acquired CoLucid, announcing the completion of the merger at the beginning of March, making it a wholly owned subsidiary [3]. The deal brings this late stage investigational medicine back under Lilly's ownership. Additional Phase III studies are planned for later in 2017, with a potential for a regulatory filing in 2018.

\section{Otsuka Pharmaceutical \& Neurovance, Inc}

Centanafadine is a novel triple reuptake inhibitor that modulates norepinephrine, dopamine and serotonin uptake, and has been under development by Neurovance, Inc. (MA, USA) as a nonstimulant medication for the treatment of attention-deficit hyperactivity disorder (ADHD) in adults and pediatric patients. A completed Phase IIb study demonstrated favorable results for a sustained release formulation of centanafadine opening a pathway to start Phase III trials during 2017 [4].

Stimulants currently used to treat ADHD, such as amphetamine derivatives, can be highly effective but can have important limitations including side effects of sleeplessness, weight loss and risk of abuse. The potential for abuse means that they are regulated as controlled substances. Existing approved nonstimulant medications, such as atomoxetine, are not controlled substances. However, nonstimulants generally have significantly lower efficacy than the stimulants. Results from a human abuse liability study suggest that centanafadine may have a lower risk of abuse compared with stimulants currently used to treat ADHD. Also at doses above the therapeutic range, an acute onset of aversion and disliking develops. This may further serve to limit abuse and may qualify centanafadine for less restrictive controlled substances scheduling.

This profile, and the ambitions of Otsuka Pharmaceutical Co. to enhance its presence in mental health and address unmet needs of patients, led to Otsuka announcing its move to acquire Neurovance and establish it as a wholly owned subsidiary on the completion of the transaction, which is expected in the second quarter of 2017 [4].

\section{Threshold pharmaceuticals \& molecular templates} Molecular Templates, Inc. has developed a technology that produces a new class of biologic drug candidates, Engineered Toxin Bodies, which have the ability to induce cellular internalization against noninternalizing receptors, the affinity of an antibody and also have a novel mechanism of action in cancer treatment, ribosome inhibition. They have announced jointly with Threshold Pharmaceuticals (CA, USA) their intention to merge to form a combined entity focused on the development of novel treatments for cancer. The transaction will also provide for a $\$ 20$ million venture capital investment in the combined company [5].

The new entity will be known as Molecular Templates, Inc. and will now be able to take lead compound MT-3724 forward through pivotal studies in non-Hodgkin's lymphoma (NHL), as well as advance evofosfamide (TH-302), previously being developed by Threshold, through a Phase I trial in combination with the immune checkpoint inhibitor ipilimumab. The new company will also have preclinical programs targeting HER2, PD-L1 and CD38.

\section{Tessa Therapeutics \& Euchloe Bio}

Tessa Therapeutics announced the acquisition of Euchloe Bio to add the latter's portfolio of immune checkpoint antagonists and immune system agonists to Tessa's capabilities in immunotherapy, including the virus-specific T-cell platform technology and nextgeneration chimeric antigen receptor technologies [6]. Tessa had already made investments in Euchloe during 2016, and Euchloe had been advancing its checkpoint inhibitor antibodies (targeting PD-1, PD-L1, CTLA4, Tim3 and Lag3) into clinical trials. The acquisition brings together technologies with research and development capabilities from the two companies to potentially build out a strong immuno-oncology pipeline against solid tumors.

\section{Arix Bioscience \& Takeda}

Arix Biosciences plc (London, UK) which is involved in the identification, financing and development of both start-up and later-stage companies across healthcare and life science sectors globally, has signed a strategic agreement with Takeda Ventures (CA, USA) to provide Takeda with access to deal flow and specialist team members to create and develop companies in partnership under a joint advisory committee [7]. Takeda is interested in opportunities in oncology and gastroenterology. 
Arix Bioscience plc, Lead Discovery Centre \& University of Leeds

Arix Bioscience has also announced an agreement with the University of Leeds (Leeds, UK) and the Lead Discovery Centre GmbH (LDC, established by Max Planck Innovation and the Max Planck Society to capitalize on the potential of research from the Max Planck Institutes and based in Dortmund, Germany) [8]. Under the agreement, Arix Bioscience will both manage and fund experimental work evolving out of fundamental discoveries in diabetes and cardiovascular medicine at the University of Leeds and LDC, focusing on antagonists of a novel metabolic disease target. This project is the first one to arise from a 2016 agreement between Arix Bioscience and LDC.

\section{PTC Therapeutics \& Marathon Pharmaceuticals}

Deflazacort (Emflaza $\left.{ }^{\mathrm{TM}}\right)$, a corticosteroid regarded as mineral sparing (compared with prednisolone), was approved (tablets and oral suspension) by US FDA in February 2017 as treatment for Duchenne muscular dystrophy (DMD), having been developed for this indication by Marathon Pharmaceuticals, LLC (IL, USA). Corticosteroid therapy is part of the standard of care in DMD. Deflazocort has been available for a number of years outside of the USA for other indications where corticosteroid therapy is appropriate, and at relatively low cost to payers which was a part of the backlash when Marathon proposed to market Emflaza at a price that would have meant that DMD treatment would cost payers $\$ 89,000$ per year for this drug alone. Consequent to this controversy, Marathon Pharmaceuticals delayed the launch of Emflaza.

PTC Therapeutics (NJ, USA), with a nearly 20-year commitment to the DMD community, has now announced their entry into an asset purchase agreement to acquire Emflaza ${ }^{\mathrm{TM}}$ from Marathon Pharmaceuticals [9] with the intention to launch the product, although at the time of writing, no commercialization plans have been shared, including the price that PTC intends to ask for the product.

\section{Vertex \& Concert Pharmaceuticals}

Adding to its portfolio of agents for the treatment of cystic fibrosis (CF), Vertex Pharmaceuticals, Inc. (MA, USA) announced that it had signed a definitive asset purchase agreement to acquire the investigational compound, CTP-656, from Concert Pharmaceuticals (MA, USA) [10]. As part of the agreement, Vertex will acquire rights to all of Concert's other CF programs.

Concert technology is based on selective incorporation of deuterium into already established compounds. This can improve their metabolic profiles, including reducing first-pass metabolism thus increasing bio- availability, and by reducing the extent of metabolism can increase in the half-life of the compound in the body [11]. CTP-656 is a deuterated analog of Vertex's already marketed CF therapeutic ivacaftor, a CF transmembrane conductance regulator potentiator, and with its modified half-life it may be useful as part of once-daily dosage treatment of a combination regimen with other CF therapeutics in the Vertex pipeline.

\section{Collaborations}

\section{Calypso Biotech \& EA Pharma}

Calypso Biotech (Plan-les-Ouates, Switzerland), focused on novel biologics as therapeutic agents in gastrointestinal (GI) disease, was spun out of Merck in 2013, funded by Merck Ventures. It has entered into a global licensing and collaboration agreement with EA Pharma Co., Ltd to develop its anti Matrix metalloproteinase-9 fully human monoclonal antibody CALY-001 [12]. Matrix metalloproteinase-9, an extracellular matrix-degrading enzyme secreted as its inactive pro-enzyme and cleaved to its active form by other proteinases, is thought to be excessively expressed and activated within inflamed GI tissue in inflammatory bowel disease.

EA Pharma Co., Ltd is a Tokyo, Japan-headquartered GI specialty pharma having a full chain of research, development, production, logistics, sales and marketing functions, created in April 2016 by the integration of the GI business units of Esai Group and Ajinomoto Group. Under the agreement, for upfront milestone and royalty payments, EA Pharma is granted an exclusive, world-wide license for the development, manufacturing and marketing of CALY-001 and both companies will collaborate on its development for inflammatory bowel disease. It may also be a therapeutic option for several oncologies, fibrosis and other inflammation indications.

\section{Vernalis plc \& Servier}

Extending an existing agreement made in 2007, Vernalis plc (Winnersh, UK) and Servier (Suresnes, France) announced a reinforcement of their partnership by entering into new 2-year oncology drug discovery collaboration on an undisclosed target [13]. The partnership will combine the Vernalis proprietary fragment and structure-based drug discovery platform with the oncology expertise at Servier. There has already been success in targeting the apoptosis inhibitory proteins Bcl-2 and Mcl-1, with compounds currently in clinical development.

\section{Michigan Medicine \& Medlmmune}

Bringing together industry and academic researchers, the University of Michigan Medical School's Michigan 
Medicine (MI, USA) and the global biologics research and development arm of AstraZeneca, MedImmune (MD, USA) has entered into a 3-year research alliance [14]. The two organizations are combining their research expertise to explore how the CNS and peripheral tissues rely on each other in diabetes, obesity and related metabolic disorders with the intent of identifying potential new therapies in these disease areas. Michigan Medicine experts in metabolism, gastroenterology and neuroendocrinology will work with MedImmune on projects in the industrial partner's cardiovascular and metabolic diseases research portfolio.

\section{AstraZeneca \& Circassia}

AstraZeneca (Cambridge, UK) announced its strategic collaboration with respiratory biopharmaceutical company Circassia (Oxford, UK) for the development and commercialization in the USA of two of its inhaled medicines for the treatment of chronic obstructive pulmonary disease [15]. These are the already approved aclidinium bromide inhalation powder (Tudorza ${ }^{\circledR}$ ) and an aclidinium bromide/formoterol fumarate dihydrate fixed-dose combination inhalation powder $\left(\right.$ Duaklir $\left.^{\circledR}\right)$, which are expected to be submitted to the FDA during 2018. Under the agreement, Circassia will lead the promotion of Tudorza in the USA and has an option to gain full commercial rights in the future. It has been granted the rights to Duaklir in the USA. AstraZeneca will receive a minority stake in Circassia and will complete ongoing development activities for the medicines and will continue to manufacture and supply both.

Heptares enters agreement with Daichi Sankyo Heptares Therapeutics (Welwyn Garden City, UK) is a clinical-stage company creating novel medicines targeting GPCRs - in particular, highly validated, yet historically undruggable, GPCRs. Its proprietary StaR (stabilized receptor) technology and structure-based drug design capabilities are being used to build a pipeline of new medicines with potential in a number of diseases, including Alzheimer's disease, schizophrenia, cancer, migraine and metabolic disease. StaR proteins are GPCRs with a small number of point mutations that greatly improve thermal stability without disrupting pharmacology. The wild-type proteins are intractable to structural studies, whereas StaR proteins allow structure determination and enable a structure-based drug design approach to GPCR drug targets [16].

Adding to its existing agreements with several other global pharmaceutical companies, Heptares has announced its entry into a drug discovery and licensing agreement with Daichi Sankyo Company, Ltd (Tokyo, Japan) focused on a single GPCR identified as playing a crucial role in relieving pain and that was nominated by the Japanese partner [17]. Daichi Sankyo will receive exclusive global rights to develop, manufacture and commercialize molecules discovered by Heptares arising from the agreement and Heptares will gain upfront payment and research funding and will be eligible for additional research, development and commercialization milestones as well as royalties from net sales of products resulting from the alliance.

\section{Allergan \& Editas Medicine}

Leber Congenital Amaurosis (LCA) is a group of inherited retinal degenerative diseases caused by mutations in at least 18 different genes, and although it is a rare disease it is the commonest cause of inherited childhood blindness (2-3 incidences per 100,000 live births worldwide). The most frequently occurring form of the disease, LCA10 (20-30\% of LCA subtypes), is caused by mutations in CEP290, hence has the potential for therapy via genome-editing approaches. Editas Medicine (MA, USA) has developed a CRISPR genomeediting platform that has generated a lead program for the potential treatment of LCA10. Allergan plc (Dublin, Ireland) has established a strategic research and development alliance with Editas Medicine to license the latter's lead genome-editing ocular program against LCA10 (currently in preclinical development) plus up to four other genome-editing eye disease programs [18]. The alliance has the potential to generate novel therapies for retinal diseases that would add to Allergan's established eye care medicines and its eye disease development programs. Under the agreement, Editas has option rights to codevelop and copromote products arising from the agreement. Allergan will undertake development and commercialization of the optioned products.

\section{Charles River Laboratories \& Chiesi Farmaceutici}

S.p.A

Building on the back of an established 6-year partnership, the early-stage contract research organization, Charles River Laboratories (MA, USA), announced an extension of its integrated drug discovery partnership with Chiesi Farmaceutici S.p.A (Parma, Italy) in the field of respiratory disease. The relationship has already generated patents and development candidates, with Charles River providing a broad range of services designed to enable selection of candidates for preclinical development by Chiesi, including medicinal chemistry, ADME/DMPK studies, safety pharmacology studies, pharmaceutics assay and models. Chiesi has an established and a development portfolio of products in asthma and chronic obstructive pulmonary disease, with its own pressurized metered-dose inhaler and dry powder inhaler technologies [19]. 


\section{PureTech Health \& Novartis}

PureTech Health plc (MA, USA) has announced a licensing and equity agreement with Novartis (Basel, Switzerland) to progress two clinical stage programs targeting the mTORC1 pathway in diseases related to immunosenescence, an age-related decline in immune function [20]. PureTech Health will develop the new selective mTORC1 product candidates in an operating subsidiary, resTORbio, with Phase IIb trials planned to commence later in 2017. Inhibition of mTORC1 has been shown to re-establish T-cell composition and function and may be of utility in addressing impaired adaptive systems and ameliorate aging-related diseases, including decline in organ function, incidence of cancer and the ability to fight infections. By targeting the underlying pathophysiology of age-associated diseases of the immune, nervous and GI systems in this way, PureTech believes they may be able to ameliorate multiple conditions with a favorable safety profile. Novartis has already explored inhibition of mTORC1 in a Phase IIa study in hundreds of elderly patients, which are to be the subject of an upcoming peer-reviewed publication.

\section{Medlmmune \& Sanofi-Pasteur}

MEDI8897, a monoclonal antibody discovered by MedImmune, neutralizes respiratory syncytial virus (RSV) by binding the RSV fusion protein expressed on virions and infected cells. RSV is among the most common causes of lower respiratory tract infections in young children and each year causes thousands of hospitalizations and outpatient visits for young children and many hundreds of thousands of hospitalizations and thousands of deaths in the elderly. MedImmune's parent company, AstraZeneca, already has the monoclonal antibody palivizumab (Synagis ${ }^{\mathrm{TM}}$ ) approved for the prevention of RSV infection in pediatric patients at high risk of severe RSV disease. Compared with palivizumab, MEDI8897 has along half-life, meaning that just a single dose (rather than monthly dosing throughout the RSV season) will be needed to afford protection for the whole season; it is currently in a Phase IIb trial in preterm infants ineligible for palivizumab [21].

The development and commercialization of MEDI8897 was the subject of an agreement announced jointly by MedImmune and Sanofi Pasteur (Lyon, France) at the beginning of March. Sanofi-Pasteur will make upfront, development milestone and sales-related milestone payments and MedImmune and AstraZeneca will lead all development activity through initial regulatory approvals. A Phase III trial in healthy fullterm and late preterm infants is planned to lead to an RSV disease prevention approach for all infants. AstraZeneca will retain manufacturing activity and SanofiPasteur will lead the commercialization activities [21].
Incyte \& Merck

Incyte Corporation (DE, USA) discovered epacadostat (INCB024360), a first-in-class, highly potent and selective oral inhibitor of the indoleamine 2,3-dioxygenase 1 (IDO1) enzyme and it is currently in development as an agent that is added to immune checkpoint inhibitors (with ongoing trials combining epacadostat with pembrolizumab, nivolumab, durvalumab or atezolizumab). IDO1 is an immunosuppressive enzyme that promotes regulatory $\mathrm{T}$-cell generation and activation and allows tumors to grow by escaping immune surveillance. Inhibition of immunomodulatory mechanisms with epacadostat combination therapy may re-establish effective antitumor immune responses and facilitate the action of the immune checkpoint inhibitor [22].

At the very end of March, Incyte and Merck (NJ, USA) - known as MSD outside the USA and Canada - announced additional details of their collaborative development program investigating the combination of epacadostat with Merck's anti-PD-1 immune checkpoint inhibitor, pembrolizumab (Keytruda ${ }^{\circledR}$ ), in patients across five tumor types: metastatic melanoma, non-small-cell lung cancer (NSCLC), bladder cancer, renal cell carcinoma and squamous cell carcinoma of the head and neck (SCCHN) [23]. The collaboration includes seven pivotal Phase III studies: one in metastatic melanoma, two in first-line NSCLC, two in bladder cancer (one first line and one second line), one in first-line renal cell carcinoma and one in first-line SCCHN. Incyte and Merck will share responsibilities for funding the studies and Merck will be responsible for conducting them.

\section{Bristol-Myers Squibb \& GRAIL, Inc.}

Bristol-Myers Squibb announced its equity investment and plans for a research collaboration with GRAIL, Inc., a Menlo Park, California-based life science company [24]. GRAIL's aim is to create a diagnostic platform to detect cancer early in asymptomatic individuals by combining high-intensity sequencing - looking at the small fragments of tumor DNA that appear in systemic circulation (circulating tumor DNA) - with modern computer science plus one of the largest genomic medicine clinical trial programs. The ability to detect cancer at its earliest stages would enable earlier intervention with targeted therapies. Through the collaboration, Bristol-Myers Squibb will be able to use GRAIL's comprehensive clinical trial databases as a rich resource in understanding tumor genomics and the two companies will collaborate to use GRAIL's analytic tools on Bristol-Myers Squibb's clinical data. This could inform research and development decisions; help advance companion diagnostics; 
and improve the selection, care and management of patients through more targeted therapies.

\section{Bristol-Myers Squibb \& CytomX Therapeutics}

An expansion of their 2014 strategic collaboration to discover novel oncology therapies was announced by Bristol-Myers Squibb and CytomX Therapeutics (CA, USA) [25], covering eight additional targets (six oncology, two nononcology) using CytomX's Probody platform beyond the four existing ones. In the existing collaboration, Bristol-Myers Squibb has progressed a CTLA-4 Probody therapeutic to IND-enabling studies stage, and three other program areas in a lead discovery/optimization phase.

The target-binding region of a Probody monoclonal antibody is masked by a peptide covering the binding site which limits interaction with healthy tissue. The proteases near tumor tissue remove the mask from the Probody therapeutic that can then bind to its target on the tumor, localizing the efficacy of a potent antibodybased therapeutic [26]. Under the expanded collaboration, Bristol-Myers Squibb will provide an upfront payment and research funding over the course of the research term, as well as future development, regulatory and sales milestones payments for each target and tiered royalties on net sales of each product commercialized.

\section{Product approvals \& other regulatory news}

\section{Ocrelizumab (Ocrevus ${ }^{\mathrm{TM}}$, Roche)}

Roche (Basel, Switzerland) announced that the FDA has approved ocrelizumab (Ocrevus ${ }^{\mathrm{TM}}$ ) for intravenous infusion, a first-in-class B-cell targeted, diseasemodifying humanized monoclonal antibody therapy for the treatment of both the relapsing-remitting and the primary progressive forms of multiple sclerosis (RRMS and PPMS, respectively) [27]. Ocrelizumab, which targets CD20-positive B cells, was studied in Phase III clinical trials for RRMS and PPMS; in the PPMS trial it reduced signs of disease activity in the brain, based on observations of lesions by MRI [27].

\section{Desmopressin acetate nasal spray (Noctiva ${ }^{\mathrm{TM}}$, Serenity Pharmaceutical)}

Based on data from two randomized, placebo-controlled trials in patients with nocturia, the FDA has approved desmopressin acetate nasal spray (Noctiva $^{\mathrm{TM}}$ ), a formulation of a peptide for nasal administration in order to achieve a systemic effect, which will be marketed by Serenity Pharmaceuticals of Milford, PA, USA [24]. In these trials desmopressin acetate nasal spray treatment led to, compared with placebo, significant reductions in the mean number of times subjects needed to pass urine in the night and a higher percentage of subjects experiencing a $50 \%$ or greater reduction in that number. The product has a risk of causing hyponatremia so caution in, or exclusion of, patients at risk of hyponatremia due to age, other medication or other diseases is required. The product was previously the subject of a global agreement for its development and commercialization between Serenity and Allergan, but the two companies have agreed to terminate this agreement and Serenity will assume responsibility for commercialization and continued development [27].

\section{Nivolumab (Opdivo TM, Bristol-Myers Squibb)} for squamous cell cancer of the head \& neck

As it continues to develop further indications for its PD-1 checkpoint inhibitor nivolumab (Opdivo ${ }^{\mathrm{TM}}$ ), Bristol-Myers Squibb has announced that the Committee for Medicinal Products for Human Use has recommended approval of this product as monotherapy for the treatment of SCCHN in adults progressing on or after platinum-based therapy [28]. This is the first Committee for Medicinal Products for Human Use recommendation of a PD-1 inhibitor for this type of treatment of SCCHN. The recommendation will now go to the European Commission, which is the authority that approves medicines for the European Union.

\section{Ribociclib (LEE011, Kisqali®) (Otsuka \& Novartis)} Ribociclib (LEE011) was discovered through a collaboration agreed in 2005 between Cambridge, UKbased Astex Pharmaceuticals, a subsidiary of Otsuka Pharmaceutical Co., Ltd and Novartis. As reported in a recent Industry Update article [29], Novartis has been progressing this compound through clinical trials. It is a selective cyclin-dependent kinase inhibitor against CDK4 and CDK6, which if overactivated can enable cancer cells divide and grow quickly.

Novartis was granted approval by the FDA for ribociclib based on clinical trials where it was studied for the treatment of hormone receptor positive, human epidermal growth factor receptor-2 negative $(\mathrm{HR}+1$ HER2-) advanced breast cancer, given as combination therapy with the aromatase inhibitor letrozole, compared with letrozole alone (a standard therapy). Progression-free survival was extended significantly for the patient group treated with the combination [30]. Astex will receive a milestone payment on the basis of this regulatory approval and will also get future royalty payments based on product sales.

\section{Osimertinib (Tagrisso ${ }^{\mathrm{TM}}$, AstraZeneca)}

AstraZeneca has announced that the FDA has granted approval to osimertinib (Tagrisso ${ }^{\mathrm{TM}}$ ) tablets. It is a third-generation, irreversible EGFR tyrosine kinase 
inhibitor (TKI). It has been approved for the treatment of patients with metastatic NSCLC and carrying the EGFR T790M mutation (as detected by an FDAapproved test) and where the disease has progressed on or after therapy with an EGFR-TKI, for example gefitinib or erlotinib. Up to two-thirds of patients develop resistance to EGFR-TKIs due to the T790M mutation. It is undergoing additional clinical trials in the adjuvant and metastatic first-line settings, including patients with CNS metastases (osimertinib has been designed to have activity in the CNS) [31].

\section{Pembrolizumab (Merck) for refractory classical Hodgkin lymphoma}

Immuno-oncology therapies continue to be developed through clinical studies in a variety of cancers and are progressing to and through regulatory approval. Merck announced that their PD-1 checkpoint inhibitor pembrolizumab has been approved by the FDA for the treatment of patients with difficult-to-treat classical Hodgkin lymphoma. Difficult-to-treat patients under this approval are those with refractory disease or those who have relapsed after three or more prior lines of therapy. The approval was granted under the FDA's accelerated approval regulations based on the observed tumor response and the durability of the responses. Under the accelerated approval regulations, the continuing approval for the indication may be contingent on verification of clinical benefit in confirmatory trials [32]. This new approval adds classical Hodgkin lymphoma to three other FDA-approved indications for pembrolizumab - melanoma, NSCLC and head and neck cancer - as the various pharmaceutical companies with checkpoint inhibitors on the market and/or under development continue to work to differentiate their product from those of their competitors.

\section{Avelumab (Bavencio ${ }^{\circledR}$, Pfizer \& Merck KGaA)}

In respect of that effort to differentiate the PD-1/PD-L1 inhibitors already marketed or just becoming commercially available, Pfizer (NY, USA) and partner Merck KGaA (Darmstadt, Germany) announced the FDA approval of their human PD-L1 antibody, avelumab $\left(\right.$ Bevecio $\left.^{\circledR}\right)$, which becomes the fourth PD-1/PD-L1 inhibitor to market as it joins the already established pembrolizumab (Merck), nivolumab (Opdivo ${ }^{\mathrm{TM}}$, Bristol-Myers Squibb) and atezolizumab (Tecentriq ${ }^{\circledR}$, Genetech/Roche). However, avelumab is currently differentiated by being the first in this class of therapeutic agents to be approved to treat a rare and aggressive form of skin cancer, metastatic Merkel cell carcinoma. Review of avelumab for this indication is also currently in progress with the EMA [33]. The efficacy and safety of avelumab was evaluated in patients with metastatic
Merkel cell carcinoma whose disease had progressed on or after chemotherapy. Avelumab was approved for this indication under the accelerated approval regulations, so the continuing approval for the indication may be contingent on verification of clinical benefit in confirmatory trials. The ongoing development programs for avelumab include nine further Phase III trials in more than 15 tumor types and more than 4000 patients.

\section{Niraparib (ZejulaTM, Tesaro)}

The poly(ADP-ribose) polymerase (PARP) inhibitors are novel, small molecule, orally administered, therapeutic agents that are progressing through Phase III clinical trials for the treatment of ovarian cancer (although other tumor types may be amenable to treatment with PARP inhibitors also, and trials with PARP inhibitors are in progress in triple negative breast cancer, NSCLC, melanoma and prostate cancer). The most recently approved of these is niraparib (Zejula ${ }^{\mathrm{TM}}$, Tesaro, Inc., MA, USA) which has just gained FDA approval, joining the already approved PARP inhibitor olaparib (Lynparza ${ }^{\mathrm{TM}}$, AstraZeneca) and rucaparib (Rubraca ${ }^{\mathrm{TM}}$, Clovis Oncology, Boulder, CO, USA). Niraparib does not require BRCA mutation or other biomarker testing to select patients when using it for the maintenance treatment of women with recurrent epithelial ovarian, fallopian tube or primary peritoneal cancer and who are in partial or complete response to platinum-based chemotherapy; this distinguishes it from the already marketed PARP inhibitors that currently have this requirement. In patients with recurrent ovarian cancer, while platinum-based chemotherapy is effective, the effectiveness diminishes over time, and progression-free survival after each subsequent treatment generally gets shorter. Niraparib therapy was demonstrated in the Phase III clinical trial that was the basis of the approval to significantly improve progression-free survival in subjects who were in a response to their most recent platinum-based chemotherapy regardless of whether they were germline $B R C A$ carriers or not [34].

\section{Safinamide (Xadago ${ }^{\circledR}$, Newron \\ Pharmaceuticals/Zambon/US WorldMeds)}

Partners Newron Pharmaceuticals S.p.A. (NJ, USA and Milan, Italy), Zambon S.p.A (Milan, Italy) and US WorldMeds, LLC (KY, USA) have announced that the FDA had approved their novel, selective MAO-B inhibitor safinamide (Xadago ${ }^{\circledR}$ ) as an add on to L-dopa therapies in the treatment of Parkinson's disease to help with the management of motor fluctuations typical of the progression of the disease and long-term treatment with L-dopa [35]. The addition of the once-daily safinamide to optimized levodopa/ 
carbidopa was demonstrated in two Phase III clinical trials to significantly increase 'on' time (normal motor functioning), decrease 'off' time (impaired motor functioning) without increases in dyskinesia, and the $\mathrm{MAO}-\mathrm{B}$ over MAO-A selectively of safinamide means that diet restrictions are not required. Safinamide is already approved in Europe and is marketed there by Zambon in 11 countries. US WorldMeds has the commercialization rights in the USA.

\section{Tofacitinib citrate (Xeljanz ${ }^{\circledR}$, Pfizer) for the treatment of moderate to severe active rheumatoid arthritis}

Pfizer has announced that its JAK1/JAK3 selective orally administered, small molecule Janus kinase inhibitor tofacitinib citrate $\left(\mathrm{Xeljanz}^{\circledR}\right)$ has received approval from the European Commission for the treatment of moderate to severe rheumatoid arthritis (RA) in adults not showing adequate response to or intolerant of one or more disease-modifying antirheumatic drugs. The JAK pathway is believed to play a role in the inflammation associated with RA. Tofacitinib citrate is approved for dosing in combination with methotrexate, or as monotherapy in case of intolerance to methotrexate or where methotrexate is inappropriate [36]; it is not recommended that it be used in combination with biologic disease-modifying antirheumatic drugs or immunosuppressants (e.g., azathioprine and ciclosporin). Additionally, the effect of tofacitinib on the ability of the immune system to fight infections means patients require testing for tuberculosis before starting treatment and need to be monitored during treatment for signs and symptoms of infections including tuberculosis. Shingles, herpes zoster, hepatitis B and hepatitis $C$ may become active because of the immune suppression caused by JAK kinase inhibition, and the risk of some cancers may be increased. However, tofacitinib is considered to be an important advance in therapy of RA, as up to a third of patients with the disease may not achieve a response with current treatments and that patients may not have a sustained response.

\section{Naldemedine (Symproic ${ }^{\oplus}$, Purdue Pharma)}

Shionogi, Inc. (Osaka, Japan and NJ, USA) and Purdue Pharma L.P. (CT, USA) have collaborated on the development of a peripherally acting $\mu$-opioid receptor antagonist, naldemedine, to counter the constipating effects of pain-relieving opioids and in late March announced that the FDA had approved it as Symproic ${ }^{\circledR}$, $0.2 \mathrm{mg}$ naldemedine once-daily tablets as treatment for opioid-induced constipation in adult patients being treated with opioids for chronic noncancer pain [37]. Opioid-induced constipation is one of the most commonly reported side effects associated with the use of opioids, including among patients with chronic, noncancer pain. It will be jointly launched by the partners and commercialized by Purdue Pharma. Naldemedine is structurally related to naltrexone and consequently has been classified as a Schedule II controlled substance in the USA. Shionogi, Inc. has submitted a petition to the US Drug Enforcement Administration for the removal of this classification, which will help with prescribing of the medicine.

\section{Dupilumab (Dupixent ${ }^{\oplus}$, Sanofi \& Regeneron)}

Dupilumab is a human monoclonal antibody designed to specifically inhibit overactive signaling of IL- 4 and IL-13, which are believed to be significantly involved in the underlying and persistent inflammation seen in atopic dermatitis (eczema). While topically applied therapies are available (e.g., orticosteroids), the approval of dupilumab by the FDA near the end of March, has made available the first and only biologic medicine that works on some of the underlying mechanisms of the disease for adults with moderate to severe atopic dermatitis inadequately controlled by topical therapies. The approval was jointly announced by Sanofi (Paris, France) and Regeneron (NY, USA), and the product will be available as a prefilled syringe, allowing selfadministration by subcutaneous injection [38]. Dupilumab was evaluated by the FDA under priority review and had been given breakthrough therapy designation, which the FDA uses to expedite the review of drugs for series or life-threatening conditions. Other than therapies for skin cancers, this was the first time that the breakthrough therapy designation had been applied to a dermatology medicine.

\section{Clinical trials}

\section{Dupilumab (Sanofi \& Regeneron)}

Data from one of the clinical trials (CHRONOS) that supported the priority review approval of dupilumab described in the previous section was presented in a late-breaking oral abstract at the AAD meeting at the beginning of the month by partners Sanofi and Regeneron [39]. The trial compared treatment with topical corticosteroids plus dupilumab against topical corticosteroids plus placebo. The new data reported at the meeting, adding information on a range of secondary end points to the solid primary end point data shared in June 2016, continued to show that in uncontrolled moderate-to-severe atopic dermatitis, dupilumab with corticosteroids significantly improved measures of overall disease compared with topical corticosteroids with the placebo injection. There were significant improvements in itch, eczema and quality of life. Data at 52 weeks of treatment were comparable with data at 
16 weeks of treatment, confirming that the drug effect persists. Additionally, the results affirm that the inhibition of IL4 and IL-13 is a valid approach to the treatment of atopic dermatitis.

\section{Nolasiban, OBE001 (ObsEva)}

ObsEva SA (Geneva, Switzerland) has announced the initiation of its Phase III clinical program to evaluate the oral oxytocin receptor antagonist nolasiban, which they had licensed from Merck-Serono in 2013, in improving pregnancy and live-birth rates in women undergoing embryo transfer as part of assisted reproduction [40]. The trial will examine the utility of a single, oral dose of $900 \mathrm{mg}$ of nolasiban before embryo transfer on either day 3 or day 5 following egg retrieval. They are seeking to confirm the increase in live-birth rates of about $10 \%$ or greater, which is regarded as a major advance for patients undergoing treatment to realize their desire to have a child. Successful assisted reproduction requires the receptivity of the uterus to accept embryo implantation, and oxytocin receptor antagonism may help by modulating uterine contractions at the time of embryo transfer, as well as improving uterine wall thickness and blood flow.

\section{Bispecific anti-IL-17A/F nanobody M1095 (Merck KGaA \& Ablynx)}

Data from a Phase Ib clinical trial of an anti-IL-17A/ IL-17F bispecific nanobody, M1095, in psoriasis were presented at the AAD meeting by Merck KGaA. Merck $\mathrm{KGaA}$ is partnered with Ablynx on the development of this agent. Ablynx is a biopharmaceutical company developing novel therapeutic proteins described as a Nanobody ${ }^{\circledR}$ which are based on single-domain antibody fragments. As such they may combine the advantages of conventional antibody drugs with some of the features of small molecule drugs. They have more than 45 programs in development in therapeutic areas including hematology, respiratory disease, oncology, immuno-oncology and inflammation and have collaborations with multiple pharmaceutical companies [41].

Merck KGaA reported a reduction in disease activity with all doses of M1095 studied. At the highest dose administered at the end of the 85-day study, all patients had a $75 \%$ reduction in disease activity and had clear/almost clear skin; $56 \%$ of patients in the highest dose group had clear skin. Onset of clinical effect was described as rapid after the first administered dose and was sustained to the completion of the study. A favorable safety and tolerability profile was noted with no treatment-related serious adverse events and no dose-dependent increase in frequency or severity of adverse events [42].

\section{MP1032 (MetrioPharm)}

MP1032 is the lead compound from a group of proprietary small molecule immune modulators developed by Zurich, Switzerland-based MetrioPharm AG that are believed to act by modulating the $\mathrm{H}_{2} \mathrm{O}_{2}$-mediated activation state of macrophages and downregulate the M1 state. It does not impact $T$ cells, and preferentially affects macrophages at sites of inflammation, which differentiates MP1032 from other immune-modulators and disease-modifying drugs, and may avoid risks associated with immunosuppressive approaches such as an increased susceptibility to infections [43]. MetrioPharm has released news of positive topline results of MP1032 in a Phase IIb study in patients with moderate-to-severe psoriasis. Based on the analysis of this data, MetrioPharm is planning to undertake a Phase IIb study with 3-months duration drug treatment and using higher doses than those employed in the announced 6-week Phase IIa study [43].

\section{Secukinumab (Cosentyx ${ }^{\oplus}$, Novartis)}

Novartis presented new data at the AAD and the Annual Maui Derm for Dermatologists meeting on its fully human-specific inhibitor of the IL-17A cytokine, secukinumab $\left(\right.$ Cosentyx $\left.^{\circledR}\right)$ [44,45], which already has approvals in Europe, Japan, Australia, USA and Canada for the treatment of moderate-to-severe plaque psoriasis and also has approvals in over 65 countries (including EU and the US) for the treatment of ankylosing spondylitis and psoriatic arthritis. The new data showed that treatment with secukinumab following relapse of disease after a treatment pause was effective, with up to $80 \%$ of patients regaining clear or almost clear skin. Of the patients that discontinued treatment, $21 \%$ maintained clear skin for up to 1-year post discontinuation of treatment and $10 \%$ maintained clear skin for up to 2 years without further treatment. Patients who had suffered longer disease duration were more likely to relapse, which suggests that early intervention in the disease can increase the likelihood of patients remaining relapse free. Novartis suggested [45] that this data may indicate that IL-17A modulation with secukinumab is not only treating disease symptoms but may be modifying the course of the disease

\section{Sarecycline (Allergan \& Paratek)}

Paratek Pharmaceuticals, Inc. (MA, USA) is a biopharmaceutical company working on innovative therapies based on tetracycline chemistry and has a novel tetracycline, sarecycline, under development in partnership with Allergan for the treatment of moderate-to-severe acne in the community setting. Sarecycline is a narrow spectrum antibiotic possessing anti-inflammatory properties. The results of two Phase III placebo-con- 
trolled trials were announced by the partners in late March, demonstrating statistically significant superiority of 12 weeks of once a day sarecycline therapy over placebo, based on a scale score and on inflammatory lesion counts. Based on the data, Allergan plans to file for regulatory approval of sarecycline in the second half of 2017; Paratek retains all ex-US rights but has not yet announced any plans for regulatory filings [46].

\section{RLX030 (serelaxin, Novartis)}

Serelaxin, a relaxin receptor agonist also known as RLX030, is a recombinant form of the vasodilatory human relaxin-2 hormone, an approximately $6.0 \mathrm{kDa}$ protein that occurs in both men and women but is present at elevated levels in pregnant women where it is thought to help the body cope with the additional cardiovascular demands during pregnancy. Serelaxin has been in a Phase III trial to evaluate its efficacy, safety and tolerability when added to standard of care in patients with acute heart failure, a life-threatening leading cause of hospitalization in patients over 65 years of age and having a high mortality rate. There have been no treatments offering improvements in the mortality rates in acute heart failure for decades. In late March, Novartis announced however that the trial did not meet its primary end points of reduction in cardiovascular death through day 180 or reduction in worsening of heart failure through day 5 [47]. Novartis has stated that they are reviewing the current data further to determine next steps for the overall program, but the announcement appears to cast some doubt over the future of serelaxin.

\section{Sacubitril/valsartan (Entresto ${ }^{\circledR}$, Novartis)}

Alongside their disappointment with serelaxin, Novartis was able to release some more positive news in the treatment of chronic heart failure at the ACC Annual Scientific Session, describing the outcome of a new posthoc analysis of a subgroup of patients in its PARDIGM-HF trial [48]. This Phase III trial had evaluated the safety and efficacy of a fixed-dose combination product of the neprilysin inhibitor sacubitril and the angiotensin receptor blocker valsartan in patients with reduced fraction heart failure (HFrEF), also referred to as systolic heart failure, compared with treatment with an ACE inhibitor, enalapril. In HFrEF, there is a reduced force of contraction by the heart and so it is less effective at pumping blood, a debilitating condition that affects half of the patients diagnosed with heart failure. The subgroup of patients in this trial analyzed by Novartis had HFrEF with diabetes. The analysis showing that treatment with the sacubitril/ valsartan fixed-dose combination product improved glycemic control compared with enalapril, producing a lowering of HbA1c levels in the diabetic patients after 1 year of therapy for heart failure, and that the effect was sustained over 3 years of study follow-up. New use of oral diabetes agents or insulin therapy was reduced. The sacubitril/valsartan fixed-dose combination does not have an approved indication for the treatment of diabetes.

\section{Rivaroxaban (Xarelto ${ }^{\circledR}$, Johnson \& Johnson \&} Bayer)

As part of its collaboration with partner Bayer, Janssen Pharmaceuticals (DC, USA), part of Johnson and Johnson, announced results at ACC from the EINSTEIN CHOICE trial, showing that patients with venous thromboembolism (VTE) taking rivaroxaban $\left(\right.$ Xarelto $\left.{ }^{\circledR}\right)$ over an extended period of time had significantly fewer recurrent blood clots compared with those taking aspirin, and with similar rates of major bleeding [49]. A third of VTE patients, which includes deep vein thrombosis and pulmonary embolism, die. All patients with VTE are at risk of having another occurrence, hence anticoagulant therapy (warfarin or a nonvitamin $\mathrm{K}$ antagonist oral anticoagulant) is initially given, and aspirin may be offered to prevent VTE recurrence once anticoagulant therapy is stopped. EINSTEIN CHOICE was undertaken to asses if rivaroxaban might be appropriate instead of aspirin for continued VTE management.

Data from the Phase II GEMINI-ACS-1 were also described. This trial compared the addition of either rivaroxaban or aspirin to a P2Y12 inhibitor (clopidogrel or ticagrelor) in the treatment of acute coronary syndrome (reduced blood flow through the coronary arteries leading to impaired cardiac muscle function or cardiac muscle death). From an evaluation of exploratory efficacy end points, rivaroxaban and aspirin were found to have similar rates of cardiovascular events in acute coronary syndrome patients, and clinically significant bleeding and expected risk of treatment, was also similar between the two treatment groups [49].

\section{Apixaban (EliquisTM, Bristol-Myers Squibb \& Pfizer)}

Partners Bristol-Myers Squibb and Pfizer presented findings at ACC from a real-world data analysis of the US Medicare database comparing the risk of stroke or systemic embolism and also the major bleeding rates in patients 65 years of age or older with nonvalvular atrial fibrillation and who had received either a direct oral anticoagulant (apixaban, dabigatran or rivaroxaban) or warfarin as treatment.

From the database analysis, Bristol-Myers Squibb and Pfizer determined that their direct oral anticoagulant, apixaban, was associated with a lower risk of 
stroke or systemic embolism, and was associated with a lower rate of major bleeding, compared with warfarin. The findings are complementary to those from the Phase III ARISTOTLE trial which supported the approval of apixaban in treatment of nonvalvular atrial fibrillation [50].

\section{Inclisiran (The Medicines Company \& Alnylam} Pharmaceuticals)

In a presentation at a late-breaking clinical trial research session at ACC, The Medicines Company and Alnylam Pharmaceuticals, Inc. announced positive results from the Phase II ORION-1 study of inclisiran for the treatment of hypercholesterolemia [51]. Inclisiran is an investigational GalNAc-conjugated RNAi therapeutic-targeting PCSK9. The carbohydrate-based GalNAC ligand conjugated on the RNAi allows for good internalization into hepatocytes, and it also permits subcutaneous administration, a benefit over prior RNAi therapeutics based on encapsulation in lipid nanoparticles, which are more challenging to prepare and must be given by intravenous infusion [52].

In the study, inclisiran showed significant and sustained reductions in LDL-C, with good safety and tolerability [51]. Additionally, the trial data indicated, and which may be consequent to its mechanism of action, that it may only be necessary to administer inclisiran every 6 months, a possible distinguishing attribute over the monoclonal antibody PCSK9 inhibitors alirocumab (Praluent ${ }^{\circledR}$, Sanofi/Regeneron) and evolocumab (Repatha ${ }^{\circledR}$, Amgen, Thousand Oaks, CA, USA), which are typically injected every few weeks.

\section{Ublituximab, TG1101 (TG Therapeutics)}

TG Therapeutics (NY, USA) has announced positive topline results from its Phase III clinical trial of its anti-CD20 monoclonal antibody ublituximab (TG1101) added to ibrutinib (Imbruvica ${ }^{\circledR}$, Pharmacyclics, CA, USA), a small molecule irreversible inhibitor of Bruton's tyrosine kinase already approved for the treatment of chronic lymphocytic leukemia. The trial compared ublituximab plus ibrutinib to ibrutinib alone in patients with previously treated high risk (17p deletion, 11q deletion or p53 mutation) chronic lymphocytic leukemia. The overall response rate in patients treated with ublituximab and ibrutinib was $80 \%$, compared with a $47 \%$ overall response rate in patients on ibrutinib alone. TG Therapeutics is planning to meet the FDA in the second half of the year to discuss an accelerated approval regulatory filing [53].

\section{Abemaciclib (Lilly)}

Eli Lilly's selective cyclin-dependent kinase inhibitor against CDK4 and CDK6, abemaciclib (LY2835219), is most active against cyclin $\mathrm{D} / \mathrm{CDK} 4$, which in breast cancer has been shown to promote phosphorylation of retinoblastoma protein $(\mathrm{Rb})$, cell proliferation and tumor growth. In hormone receptor positive breast cancer cell lines, abemaciclib reduced phosphorylation of $\mathrm{Rb}$, inducing cell cycle arrest. The company has announced that, in a Phase III study of abemaciclib in combination with the selective estrogen receptor downregulator fulvestrant $\left(\right.$ Faslodex $^{\circledR}$, AstraZeneca) in women with HR+/HER2- advanced breast cancer who have relapsed or progressed after endocrine therapy, the trial met its primary end point of progression-free survival, with a statistically significant improvement in patients on abemaciclib plus fulvestrant compared with patients on fulvestrant alone [54]. The study will be the basis of a regulatory filing with the FDA in the third quarter of this year, following the prior submission of an application for the approval of abemaciclib as monotherapy. Eli Lilly has several additional trials of abemaciclib in breast cancer as part of other treatment regimens in HR+/HER2- and HR+/HER2+ disease, and a trial ongoing in lung cancer.

\section{Tezacaftor/Ivacaftor (Vertex)}

The administration of two agents affecting CFTR protein processing and addressing specific, different defects in that processing leads to better management of $\mathrm{CF}$, as demonstrated with the results from two Phase III studies completed by Vertex Pharmaceuticals (MA, USA) investigating the addition of the investigation drug tezacaftor, which works on the defect caused by the F508 del-CTFR mutation, to the already approved ivacaftor $\left(\right.$ Kalydeco $\left.{ }^{\circledR}\right)$, which is appropriate for patients with the G551D, G1244E, G1349D, G178R, G551S, S1251N, S1255P, S549N, S549R or R117H mutations. The studies met their primary end points of improvements in lung function as demonstrated by the percent predicted forced expiratory volume in one second $\left(\mathrm{pFEV}_{1}\right)$ measurements. FDA and EMA regulatory filings are planned for the third quarter of 2017 for use of the combination tezacaftor/ivacaftor treatment of patients 12 years of age and older with two copies of the F508del mutation and patients with one mutation that results in residual CFTR function and the F508del mutation [55].

\section{GLPG3067 (Galapagos)}

Galapagos (Mechelen, Belgium) entered into an alliance with AbbVie in 2013 to discover, develop and commercialize novel therapies in CF. The aim of their collaboration is to develop a triple CFTR combination therapy to address $90 \%$ of CF patients. Consistent restoration of healthy activity levels of chloride 
transport in in vitro assays with human bronchial epithelial cells of patients with the F508del mutation have been shown with triple combinations of compounds in the portfolio. Galapagos has announced that a Phase I study of the safety, tolerability and pharmacokinetics of its novel potentiator GLPG3067 has been initiated in healthy volunteers. This builds ongoing phase I studies of potentiator GLGP2451 dosed with corrector GLPG2222. A third compound, corrector GLPG2737, is also planned for Phase I evaluation during the first half of 2017 to prepare for a triple combination study by adding this agent to GLGP2451 and GLPG2222, with a plan to initiate a triple combination study in patients in mid 2017 [56].

\section{Voclosporin (Aurinia)}

Lupus nephritis, a serious progression of systemic lupus erythematosus, can lead to kidney damage if not controlled and is potentially a life-threatening condition. Aurinia Pharmaceuticals, Inc. (Victoria, BC, Canada) has been developing voclosporin, a novel, immunosuppressant calcineurin inhibitor derived from cyclosporine, which has greater potency, a more predictable pharmacokinetic/pharmacodynamic relationship and, as a consequence, potential for flat dosing compared with the parent compound. It is being explored in the treatment of lupus nephritis as an add on to standard-of-care treatment (mycophenolate mofentil) and they have released the news that the top-line results from its Phase IIb trial which showed that at 48 weeks voclosporin therapy met the complete and partial remission end points, with greater remission compared with control. This outcome included the achievement of the highest complete remission rate in any global lupus nephritis study. Phase III trials for voclosporin are now being anticipated [57].

\section{References}

$1 \quad$ MergerMarket. Trend Report Q1 2017. www.mergermarket.com/pdf/MergermarketTrendReport. Q12017.Pharma,Medical,Biotech.pdf

2 Johnson \& Johnson publishes interim result for Actelion tender offer and declares the tender offer successful. www.jnj.com/media-center/press-releases/johnson-johnsonpublishes-interim-result-for-actelion-tender-offer-anddeclares-the-tender-offer-successful

3 Lilly Completes Acquisition of CoLucid Pharmaceuticals. https://investor.lilly.com/releasedetail. cfm?ReleaseID $=1015163$

4 Otsuka Pharmaceutical to acquire Neurovance, Inc. Acquisition of clinical-stage company in ADHD widens Otsuka's presence in mental health.

\section{NKTR-181 (Nektar Therapeutics)}

Nektar Therapeutics (CA, USA) has announced positive results from a Phase III trial of its novel, selective and long acting, full $\mu$-opioid agonist NKTR-181, which was designed to provide potent pain relief without the high level of euphoria seen with standard opioids, and that can lead to abuse and addiction. Based on preclinical data, NKTR-181 has low permeability across the blood-brain barrier which slows its rate of entry into the brain and so attenuates the dopamine release that underlies euphoria. In the Phase III SUMMIT-07 study, compared with the placebo, NKTR181 provided significantly improved chronic back-pain relief. Patients on NKTR-181 also reported that their quality of life and general overall status as 'improved' or 'very much improved', which was also favorable when compared with feedback from the patients treated with the placebo. A prior study demonstrated NKTR-181 to have similar abuse potential to placebo [58]. Additional Phase III studies are ongoing; the FDA has granted fast track designation to NKTR-181 for the treatment of moderate-to-severe chronic pain.

\section{Financial \& competing interests disclosure}

The author is a former employee and a current shareholder of Bristol-Myers Squibb, which has active research programs and marketed medicines in some of the therapeutic areas covered in this article and some products are mentioned by name.

Apart from those already disclosed, the author has no other relevant affiliations or financial involvement with any organization or entity with a financial interest in or financial conflict with the subject matter or materials discussed in the manuscript. This includes employment, consultancies, honoraria, stock ownership or options, expert testimony, grants or patents received or pending, or royalties.

No writing assistance was utilized in the production of this manuscript.

www.otsuka.co.jp/en/company/release/detail.

php?id $=3240 \&$ date $=2017-03-03$

5 Threshold Pharmaceuticals and Molecular Templates agree to combine.

http://investor.thresholdpharm.com/releasedetail. cfm?ReleaseID =1017826

6 Tessa Therapeutics announces acquisition of Euchloe Bio. www.tessatherapeutics.com/news-2017/tessa-therapeuticsannounces-acquisition-of-euchloe-bio

7 Arix Bioscience plc signs strategic agreement with Takeda. https://arixbioscience.com/news-release/arix-bioscience-plcsigns-strategic-agreement-takeda

8 Arix Bioscience plc, LDC and University of Leeds collaborate to develop novel therapeutics for metabolic diseases. https://arixbioscience.com/news-release/arix-bioscience-plcldc-university-leeds-collaborate-develop-novel-therapeutics- 
metabolic-diseases

9

Therapeutics announces agreement to acquire Emflaza for the treatment of Duchenne muscular dystrophy in US. http://ir.ptcbio.com/releasedetail.cfm?ReleaseID =1017648

10 Vertex to acquire CTP-656 from Concert Pharmaceuticals for the treatment of cystic fibrosis.

http://investors.vrtx.com/releasedetail. cfm? ReleaseID $=1015937$

11 Concert Pharmaceuticals. DCE Platform ${ }^{\circledR}$ - More Efficient and Less Expensive.

www.concertpharma.com/technology-overview/dceplatform/

12 Calypso Biotech licenses CALY-001 best-in-class antiMMP-9 antibody to EA Pharma. www.calypsobiotech.com/ site/en/news-and-events/press-release

13 Vernalis plc and Servier enter into a new drug discovery collaboration strengthening their existing partnership. www.vernalis.com/media-centre/latest-releases/740vernalis-plc-and-servier-enter-into-a-new-drug-discoverycollaboration-strengthening-their-existing-partnership

14 Michigan Medicine and MedImmune partner in diabetes and metabolic disease research. www.uofmhealth.org/news/archive/201703/michiganmedicine-and-medimmune-partner-diabetes-and

15 AstraZeneca and Circassia enter strategic collaboration in respiratory disease.

www.astrazeneca.com/content/astraz/media-centre/pressreleases/2017/astrazeneca-and-circassia-enter-strategiccollaboration-in-respiratory-disease-17032017.html

16 Heptares. GPCR Drug Discovery - Platform. www.heptares.com/platform/

17 Heptares enters agreement with Daiichi Sankyo to discover and develop novel, small molecules for the treatment of pain. www.heptares.com/news/297/74/Heptares-entersAgreement-with-Daiichi-Sankyo-to-Discover-and-DevelopNovel-Small-Molecules-for-the-Treatment-of-Pain.html

18 Allergan and Editas Medicine enter into strategic R\&D alliance to discover and develop CRISPR genome editing medicines for eye diseases.

http://ir.editasmedicine.com/phoenix.

zhtml?c=254265 \&p =irol-newsArticle \&ID = 2253717

19 Charles River Laboratories and Chiesi Farmaceutici SpA announce extension of integrated respiratory drug discovery program.

http://ir.criver.com/phoenix.zhtml?c=121668\&p=RssLanding \& cat $=$ news $\&$ id $=2253376$

20 PureTech Health announces licensing and equity agreement with Novartis to advance clinical stage mTORC1 programmes. http://puretechhealth.com/news.php

21 MedImmune and Sanofi Pasteur form alliance to develop and commercialise potential next-generation respiratory syncytial virus antibody MEDI8897.

www.astrazeneca.com/content/astraz/media-centre/pressreleases $/ 2017 /$ medimmune-and-sanofi-pasteur-form-allianceto-develop-and-commercialise-potential-next-generationrespiratory-syncytial-virus-antibody-medi8897-030317.html
22 Incyte Corporation: What we do. www.incyte.com/what-we-do/what-we-do.aspx

23 Incyte and Merck provide additional details on previously announced collaboration investigating epacadostat and Keytruda $^{\circledR}$ (pembrolizumab). www.mrknewsroom.com/news-release/incyte-andmerck-provide-additional-details-previously-announcedcollaboration-investig

24 Bristol-Myers Squibb expands focus on precision medicine with investment and planned collaboration with GRAIL on blood-based cancer screening. https://news.bms.com/press-release/partnering-news/bristolmyers-squibb-expands-focus-precision-medicine-investmentand-p

25 Bristol-Myers Squibb and CytomX Therapeutics extend worldwide collaboration to discover Probody ${ }^{\text {TM }}$ therapeutics for the treatment of cancer and other diseases.

https://news.bms.com/press-release/partnering-news/bristolmyers-squibb-and-cytomx-therapeutics-extend-worldwidecollabor

26 FDA approves Roche's OCREVUS ${ }^{\text {TM }}$ (ocrelizumab) for relapsing and primary progressive forms of multiple sclerosis. www.roche.com/media/store/releases/med-cor-2017-03-29. htm

27 U.S. FDA approves Serenity Pharmaceutical's Noctiva ${ }^{\mathrm{TM}}$ (desmopressin acetate) - the first drug approved for the treatment of nocturia.

http://serenitypharma.com/noctiva/\#sthash.5wEOUonu.dpbs

28 Bristol-Myers Squibb receives positive CHMP opinion recommending Opdivo (nivolumab) for the treatment of squamous cell cancer of the head and neck in adults progressing on or after platinum-based therapy. https://news.bms.com/press-release/bristolmyers/bristolmyers-squibb-receives-positive-chmp-opinion-recommendingopdivo-n

29 Timmins P. An industry update: what are the latest developments in the field of therapeutic delivery? Industry Update: $1^{\text {st }}$ December to $31^{\text {st }}$ December 2016. Ther. Deliv. 8, 185-199 (2017)

30 Ribociclib (LEE011) receives regulatory approval in U.S. as a first-line treatment for HR+/HER2- advanced breast cancer with an aromatase inhibitor. www.otsuka.co.jp/en/company/release/detail. php? id $=3248 \&$ date $=2017-03-23$

31 Tagrisso (osimertinib) receives US FDA full approval. www.astrazeneca.com/content/astraz/media-centre/pressreleases/2017/tagrisso-osimertinib-receives-us-fda-fullapproval-31032017.html

32 FDA Approves Merck's Keytruda ${ }^{\circledR}$ (pembrolizumab) for adult and pediatric patients with classical Hodgkin lymphoma (cHL) refractory to treatment, or who have relapsed after three or more prior lines of therapy. www.mrknewsroom.com/news-release/corporate-news/ fda-approves-mercks-keytruda-pembrolizumab-adult-andpediatric-patients-

33 FDA grants approval for Bavecio ${ }^{\circledR}$ (avelumab), the first immunotherapy approved for metastatic Merkel cell carcinoma. 
www.pfizer.com/news/press-release/press-release-detail/ fda_grants_approval_for_bavencio_avelumab_the_first_ immunotherapy_approved_for_metastatic_merkel_cell_ carcinoma

34 Tesaro announces U.S. FDA approval of Zejula ${ }^{\mathrm{TM}}$ (niraparib) for women with recurrent ovarian cancer. http://ir.tesarobio.com/releasedetail. cfm?ReleaseID $=1018920$

35 FDA Approves Xadago ${ }^{\circledR}$ (safinamide) for Parkinson’s disease (PD) patients. www.newron.com/en

$36 \mathrm{Xeljanz}^{\circledast}$ (tofacitinib citrate) receives Marketing Authorisation in the European Union for the treatment of moderate to severe active rheumatoid arthritis (RA). www.pfizer.com/news/press-release/press-release-detail/ xeljanz_tofacitinib_citrate_receives_marketing _ authorisation_in_the_european_union_for_the_treatment of_moderate_to_severe_active_rheumatoid_arthritis_ra

37 FDA approves Symproic ${ }^{\circledR}$ (naldemedine) once-daily tablets C-II for the treatment of opioid-induced constipation in adults with chronic non-cancer pain.

www.purduepharma.com/news-media/2017/03/fdaapproves-symproic-naldemedine-once-daily-tablets-c-ii-forthe-treatment-of-opioid-induced-constipation-in-adultswith-chronic-non-cancer-pain

38 Sanofi and Regeneron announce FDA approval of Dupixent ${ }^{\circledR}$ (dupilumab), the first targeted biologic therapy for adults with moderate-to-severe atopic dermatitis. http://mediaroom.sanofi.com/?p=59316

39 Sanofi and Regeneron announce presentation of positive data from long-term pivotal Phase 3 CHRONOS study of Dupixent ${ }^{\circledR}$ (dupilumab) in moderate-to-severe atopic dermatitis. http://mediaroom.sanofi.com/?p=53634

40 ObsEva starts Phase 3 clinical program for nolasiban in ART. www.obseva.com/news/obseva-starts-phase-3-clinicalprogram-for-nolasiban-in-art

41 Ablynx's partner, Merck KGaA, has presented new data from a Phase Ib psoriasis study of the bi-specific anti-IL-17A/F Nanobody at the annual AAD conference. www.ablynx.com/news/press-releases

42 Safety and efficacy of multiple ascending doses of subcutaneous M1095, an anti-interleukin-17A/F bispecific Nanobody ${ }^{\circledR}$, in patients with moderate-to-severe psoriasis. www.ablynx.com/uploads/data/files/170301_m1095_ psoriasis_003\%20for\%20aad2017.pdf

43 MetrioPharm announces positive top line results from a Phase IIa study in moderate-to-severe psoriasis with its oral lead compound MP1032. www.metriopharm.com/en/news/2017/Announces-PositiveTop-Line-Results-from-a-Phase-IIa-Study.html

44 Novartis' Cosentyx shows almost all psoriasis patients rapidly regain skin clearance following a treatment pause. www.novartis.com/news/media-releases/novartis-cosentyxshows-almost-all-psoriasis-patients-rapidly-regain-skin

45 Novartis' Cosentyx is first and only IL-17A inhibitor to potentially modify the course of psoriasis. www.novartis.com/news/media-releases/novartis-cosentyx- first-and-only-il-17a-inhibitor-potentially-modify-course

46 Allergan and Paratek announce positive results from two Phase 3 trials of sarecycline for the treatment of moderate to severe acne. www.allergan.com/investors/news/thomson-reuters/allerganand-paratek-announce-positive-results-fro

47 Novartis provides update on Phase III study of RLX030 (serelaxin) in patients with acute heart failure. www.novartis.com/news/media-releases/novartis-providesupdate-phase-iii-study-rlx030-serelaxin-patients-acute-heart

48 New analysis shows Entresto improves glycemic control in reduced ejection fraction heart failure patients with diabetes Novartis.

www.novartis.com/news/media-releases/new-analysisshows-novartis-entresto-improves-glycemic-control-reducedejection

49 New Phase 3 study finds Xarelto ${ }^{\circledR}$ to be superior to aspirin for long-term prevention of recurrent blood clots without observing any significant increase in major bleeding in patients with venous thromboembolism. www.jnj.com/media-center/press-releases/ new-phase-3-study-finds-xarelto-to-be-superiorto-aspirin-for-long-term-prevention-of-recurrentblood-clots-without-observing-any-significant-increase-inmajor-bleeding-in-patients-with-venous-thromboembolism

50 Bristol-Myers Squibb and Pfizer present large real-world observational analysis of the effectiveness and safety of direct oral anticoagulants compared to warfarin in patients with non-valvular atrial fibrillation.

https://news.bms.com/press-release/acc17/bristol-myerssquibb-and-pfizer-present-large-real-world-observationalanalysis-

51 The Medicines Company and Alnylam Pharmaceuticals report positive final results from ORION-1 Phase II study of inclisiran.

www.themedicinescompany.com/investors/news/medicinescompany-and-alnylam-pharmaceuticals-report-positive-finalresults-orion-1

52 Alnylam Pharmaceuticals. Key Scientific Data on Enhanced Stabilization Chemistry (ESC)-GalNAc-Conjugate Technology. www.alnylam.com/capella/presentations/esc-galnacconjugates-tides $2014 /$

53 TG Therapeutics announces positive topline data from Phase 3 GENUINE study of TG-1101 in combination with ibrutinib in patients with high risk chronic lymphocytic leukemia (CLL).

http://ir.tgtherapeutics.com/releasedetail. cfm?ReleaseID $=1015939$

54 Lilly announces Phase 3 MONARCH 2 breast cancer study of abemaciclib met primary end point of progression-free survival. https://investor.lilly.com/releasedetail. cfm? ReleaseID $=1017952$

55 Two Phase 3 studies of the tezacaftor/ivacaftor combination treatment met primary endpoints with statistically significant improvements in lung function (FEV1) in people with cystic fibrosis. http://investors.vrtx.com/releasedetail. 
cfm?ReleaseID $=1019156$

56 Galapagos initiates Phase 1 study with novel CF potentiator GLPG3067.

www.glpg.com/press-releases

57 Aurinia announces voclosporin meets 48-week remission endpoints, achieving highest complete remission rate of any global lupus nephritis study. http://ir.auriniapharma.com/press-releases/detail/73/ aurinia-announces-voclosporin-meets-48-week-remission

58 NKTR-181 meets primary and secondary end points in Phase 3 SUMMIT-07 study in chronic pain.

http://ir.nektar.com/releasedetail.cfm?ReleaseID =1017974 
